\title{
Teasing Apart Translational and Transcriptional Components of Stochastic Variations in Eukaryotic Gene Expression
}

\author{
Raheleh Salari $^{19 \alpha}$, Damian Wojtowicz ${ }^{1,29}$, Jie Zheng ${ }^{39}$, David Levens ${ }^{4}$, Yitzhak Pilpel ${ }^{5}$, \\ Teresa M. Przytycka ${ }^{1 *}$
}

1 National Center for Biotechnology Information, National Library of Medicine, Bethesda, Maryland, United States of America, $\mathbf{2}$ Institute of Informatics, University of Warsaw, Warsaw, Poland, 3 Bioinformatics Research Centre (BIRC), School of Computer Engineering, Nanyang Technological University, Singapore, $\mathbf{4}$ Laboratory of Pathology, National Cancer Institute, Bethesda, Maryland, United States of America, $\mathbf{5}$ Department of Molecular Genetics, Weizmann Institute of Science, Rehovot, Israel

\begin{abstract}
The intrinsic stochasticity of gene expression leads to cell-to-cell variations, noise, in protein abundance. Several processes, including transcription, translation, and degradation of mRNA and proteins, can contribute to these variations. Recent single cell analyses of gene expression in yeast have uncovered a general trend where expression noise scales with protein abundance. This trend is consistent with a stochastic model of gene expression where mRNA copy number follows the random birth and death process. However, some deviations from this basic trend have also been observed, prompting questions about the contribution of gene-specific features to such deviations. For example, recent studies have pointed to the TATA box as a sequence feature that can influence expression noise by facilitating expression bursts. Transcriptionoriginated noise can be potentially further amplified in translation. Therefore, we asked the question of to what extent sequence features known or postulated to accompany translation efficiency can also be associated with increase in noise strength and, on average, how such increase compares to the amplification associated with the TATA box. Untangling different components of expression noise is highly nontrivial, as they may be gene or gene-module specific. In particular, focusing on codon usage as one of the sequence features associated with efficient translation, we found that ribosomal genes display a different relationship between expression noise and codon usage as compared to other genes. Within nonribosomal genes we found that sequence high codon usage is correlated with increased noise relative to the average noise of proteins with the same abundance. Interestingly, by projecting the data on a theoretical model of gene expression, we found that the amplification of noise strength associated with codon usage is comparable to that of the TATA box, suggesting that the effect of translation on noise in eukaryotic gene expression might be more prominent than previously appreciated.
\end{abstract}

Citation: Salari R, Wojtowicz D, Zheng J, Levens D, Pilpel Y, et al. (2012) Teasing Apart Translational and Transcriptional Components of Stochastic Variations in Eukaryotic Gene Expression. PLoS Comput Biol 8(8): e1002644. doi:10.1371/journal.pcbi.1002644

Editor: Sarah A. Teichmann, MRC Laboratory of Molecular Biology, United Kingdom

Received February 4, 2012; Accepted June 18, 2012; Published August 30, 2012

This is an open-access article, free of all copyright, and may be freely reproduced, distributed, transmitted, modified, built upon, or otherwise used by anyone for any lawful purpose. The work is made available under the Creative Commons CCO public domain dedication.

Funding: The research was supported in part by the Intramural Program of National Institutes of Health NLM (RS, JZ, DW, TMP) and NCl, CCR (DL), as well as in part by a grant from the Polish Ministry of Science and Higher Education (NN301065236) to DW. YP is supported by an "Ideas" grant of the European Research Council and the Ben May Foundation. JZ was also supported in part by start-up grant (M4080108.020) at Nanyang Technological University, Singapore. The funders had no role in study design, data collection and analysis, decision to publish, or preparation of the manuscript.

Competing Interests: The authors have declared that no competing interests exist.

*E-mail: przytyck@ncbi.nlm.nih.gov

9 These authors contributed equally to this work.

a Current address: Department of Computer Science, Stanford University, Stanford, California, United States of America

\section{Introduction}

The stochastic nature of gene expression promotes cell-to-cell differences in protein level, usually referred to as noise $[1,2,3]$. Recent studies, both experimental and computational, have revealed that such cell-to-cell variability can be both disadvantageous $[4,5,6,7,8]$, as variations in protein level might negatively affect the precision of signaling and regulation, and advantageous $[9,10,11,12,13,14]$, by enabling heterogeneous stress-response programs to environmental changes [10]. Expression noise has also been proposed to have an important impact on gene evolution $[6,15,16]$. These diverse roles are expected to be accompanied by complex and heterogeneous modes of noise regulation. In addition, feedback loops and other network motifs might be utilized to regulate noise $[17,18,19,20]$ or propagate it through regulatory networks $[19,21,22]$, adding to the overall complexity.

The sources of variation in gene expression in an isogenic cell population are typically divided into two basic groups: (i) the intrinsic noise attributed to the inherent stochasticity of expression processes, and (ii) the extrinsic noise resulting from variation in cell state related to cell-cycle progression, cell size, subtle environmental differences, and other stochastic events that are external to the system - in this case external to the process of expression of an individual gene $[1,23,24,25,26,27,28]$. Several stochastic processes including transcription, translation, and mRNA and protein degradation can contribute to the intrinsic noise [2]. The relative contribution of these components is gene or gene-module specific. Basic factors can be gleaned from correlations between noise level 


\section{Author Summary}

The stochastic nature of gene expression leads to cell-tocell differences in protein level referred to as noise. Expression noise can be disadvantageous, by affecting the precision of biological functions, but it may also be advantageous by enabling heterogeneous stress-response programs to environmental changes. Therefore various genes and gene groups might display various levels of expression noise. Importantly, gene expression is a multistep process and the stochasticity of its individual steps, including transcription and translation, contributes to the resulting variability. Recent single cell analyses of gene expression in yeast have confirmed the theoretically predicted general trend where expression noise scales with protein abundance. However, some deviations from this basic trend have also been observed, prompting questions about the contribution of gene-specific features to such deviations. Accounting for noise heterogeneity in different gene groups, we revealed a clear relationship between noise and translation-related genomic features, specifically codon usage and $5^{\prime}$ UTR secondary structure. Our results suggest that the effect of translation on these deviations might be more prominent than previously appreciated, and provide important clues towards understanding expression stochasticity in yeast.

and gene characteristics such as promoter structure, gene function, essentiality, chromatin density, and similar features [29]. In the context of the prokaryote $B$. subtilis, it has been observed that the predominant source of phenotypic noise strength is translational efficiency [30]. It has been proposed that in prokaryotes, low transcription but high translation rates produce protein bursts leading to strong fluctuations in the protein level [17,30]. In contrast, noise in eukaryotic gene expression is assumed to be predominantly influenced by the dynamics of transcription $[29,31,32]$, in particular transcription bursts [33,34,35]. Transcription bursts are not unique to eukaryotes and also have a clear impact on prokaryotic noise [36],[37]. Similarly, translation dynamics is expected to have an impact on eukaryotic gene expression. Along this line, Blake et al. demonstrated experimentally that codon usage can impact noise strength in eukaryotic gene expression and proposed that increased translational efficiency might have a substantial effect when coupled with a noisy transcriptional state [31]. Furthermore, a recent analysis of data collected by Bar-Even et al. showed some tendency for efficiently translated genes to have increased noise [38].

Single-cell analyses of gene expression in yeast provided an important step towards understanding noise etiology and demonstrated a general trend where expression noise scales with protein abundance [29]. This trend suggests that expression of most genes follows roughly a random stochastic process. Importantly, there are some deviations from this basic trend, indicating that gene specific factors might be altering this general behavior. Newman et al. measured these deviations with the DM measure, defined as the difference of the gene specific noise and the median noise for proteins with the same abundance, as estimated from the trend line for the relation between noise and abundance. We use the term noise differential to denote such deviation of the noise of an individual gene from the average trend, and thus DM is a measure of noise differential. Studies by Newman et al. [29] have uncovered a highly significant correlation of noise differential with several transcription regulation features, including the presence of a
TATA box, but did not reveal such highly significant correlation with codon usage, the hallmark of translation efficiency [39,40,41]. However, untangling different components of expression noise is highly nontrivial. Intrinsic and extrinsic fluctuations can be separated experimentally by utilizing dual reporter measurements $[1,24]$, but experimental separation of transcriptional and translational components would additionally require single-cell measurements of both mRNA and protein copy numbers simultaneously [42].

To complement these studies, we used computational means to investigate the question of to what extent sequence features known or postulated to accompany translation efficiency can also be associated with noise differential. Specifically, we considered codon usage, as measured by the tRNA adaptation index (tAI), and $5^{\prime}$ UTR structure. High tAI is postulated to contribute to efficient translation elongation, while low secondary structure at the $5^{\prime}$ UTR has been shown to negatively correlate with ribosomal density $[43,44,45,46,47,48]$. Thus, these two features may potentially correlate with amplification of the strength of transcription noise and noise differential.

We observed that ribosomal proteins display a different relationship between expression noise and codon usage as compared to other proteins. Focusing on nonribosomal proteins, we found that the above-mentioned features indeed have significant associations with noise differential. Among these features, the statistical significance of the association with tRNA adaptation index is the highest. We then used a theoretical noise expression model to decompose the protein abundance associated noise strength into two components: noise associated with transcription (represented by the presence of a TATA box) and noise putatively associated with translation (represented by high tAI), while controlling for the protein abundance. Strikingly, we found that the amplification of noise strength associated with high tRNA adaptation index is comparable to the amplification of noise strength associated with the presence of a TATA box. The noise factoring strategy that we introduced here for the purpose of uncovering relative interplay between these two factors is general and can be readily applied to tease apart other contributions of interest.

\section{Results}

Noise is defined as the coefficient of variation $C V=\sigma / \mu$, where $\mu$ is the mean and $\sigma^{2}$ is the variance of experimental measurements. Recent single-cell studies of gene expression in yeast have uncovered a general trend where the squared coefficient of variation is inversely proportional to protein abundance [29,32]. Supporting this understanding, Bar-Even et al. provided a theoretical argument for the hypothesis that expression noise results from a stochastic process where mRNA copy number follows the random birth and death process. Given this principal scaling property, attention has been turned towards uncovering systematic deviations from this abundance-related trend and correlating such deviations with specific gene features. Here we use the data gathered in the experiment of Newman et al., where the trend line was only paralleling the Poissonian process for low to moderate expression levels. Using a two-dye experiment on a sample of highly expressed proteins, the experimenters demonstrated that these deviations from the Poissonian process for high expression levels are caused by extrinsic noise. Newman et al. accounted for this effect by introducing the DM measure (defined above).

\section{Heterogeneity of noise properties in different gene groups}

Given that translation efficiency has been found to impact cellto-cell noise in prokaryotic organisms [30] and that translation 
efficiency has been demonstrated to have the potential to amplify transcription noise in eukaryotic cells [31], the low statistical significance of the correlation between codon usage and noise in Newman and colleagues' large-scale yeast study [29] was to some extent unexpected. Remarkably, we observed that the distribution of codon usage (as measured by tRNA adaptation index [49]) has a long tail (Figure 1a). Removing this tail at a wide range of cut-off values increases the significance of the Spearman correlation between tAI and DM (Figure la inset). We noticed that the genes at the tail of the tAI distribution are highly enriched in ribosomal genes - 98 out of 153 genes with tAI above 0.55 are ribosomal (binomial test, $p$-value $<\mathrm{e}-74$ ). Comparing all ribosomal and nonribosomal genes, we found that these two groups have different distributions of noise differentials $(\mathrm{DM})$ - the ribosomal group is significantly less noisy than the remaining genes (Figures $1 \mathrm{~b}$ and 1c; Wilcoxon rank-sum test, $p$-values $<\mathrm{e}-4.7$ and e-24.4 for YEPD and SD, respectively); the difference is also statistically significant for equal sample sizes (Supplementary Table S1). In addition, the correlation between DM and tAI in ribosomal group was negative (Figure 1d; Spearman correlation $-0.4, p$-values $<\mathrm{e}-7$ for both YEPD and SD). In contrast, we observed a highly significant positive correlation between noise differential and tRNA adaptation index in the group of nonribosomal genes, suggesting a robust contribution of the translation process to expression noise in this group of genes (Figure 1d; Spearman correlation, $p$-values $<$ e-11.1 and e-9.6 for YEPD and SD, respectively). Given this different relation between noise differential and codon usage for these two groups of genes, we removed all ribosomal genes (see Materials and Methods) from further analyses and focused only on nonribosomal genes.

Additionally, we combined the measurements from both growth media and subdivided the nonribosomal genes into three groups according to noise level: low, medium and high noise genes (see Materials and Methods for precise description of the grouping). Figure 2a shows statistically significant differences in tRNA adaptation index between these three noise differential levels (Wilcoxon rank-sum test, all $p$-values $<\mathrm{e}-3.9$ ).

\section{Variations in mRNA secondary structure correlate with variations in noise differential}

The structure of messenger RNA is known to affect translation efficiency $[43,44,45,46,47,48]$. A low level of secondary structure at the 5' UTR of mRNA correlates with increased ribosomal density, leading to an increased translation rate $[43,44,45,46,47,48,50,51]$. More importantly, simultaneous translation of several protein molecules from the same mRNA molecule leads to a deviation from the Poissonian model of gene expression (see also Fraser et al. [4]). Therefore, we conjectured that the level of secondary structure at the 5' UTR of mRNA might impact expression noise. To test this hypothesis we computed, for each gene in the nonribosomal group and each position within the gene, the gene base pairing probability in RNA structure (Materials and Methods). Indeed, we observed statistically significant inverse correlation between $5^{\prime}$ UTR secondary structure and noise differential (Figure 2b; Spearman correlation, $p$-values $<\mathrm{e}-3.2$ and e-8.2 for YEPD and SD, respectively). Using the same subdivision of the genes into three groups as for the codon usage above, we observed that the noisiest genes were characterized by the lowest base pairing probability in the 5' UTR region, while the least noisy genes had the most structured 5' UTR. The differences between the highly noisy group and remaining two groups are also statistically significant (Figure 2b; Wilcoxon rank-sum test, $p$ values $<$ e-9.4 and e-6.1 for comparison with low and medium noise genes, respectively; Supplementary Table S1 for equal size set sampling).

Potentially, if a gene is optimized for rapid but noisy expression then optimization of codon usage is likely to be accompanied by other efficiency boosting features such as unstructured 5' UTR. Indeed, there is a highly statistically significant inverse correlation between $5^{\prime}$ UTR structure and tAI (Spearman correlation -0.2; $p$-value $<\mathrm{e}-22)$. Partial correlation analysis indicates that these correlations remain statistically significant when controlling for the third factor (Supplementary Table S2). The correlation of 5' UTR with DM in YEPD was the weakest and after controlling for tAI or TATA was only marginally significant $(p$-value $=0.0366$ and $p$ value $=0.0134$ respectively). Therefore we did not include 5 ' UTR in our next analysis, where we used a theoretical noise expression model to decompose the gene-specific amplification of noise strength into a transcriptional component attributed to the TATA box and a putative translational component attributed to tAI.

\section{Decomposing noise strength amplification into TATA and $\mathrm{tAl}$ associated components}

Given the above-demonstrated relationship between the sequence features associated with translational efficiency and noise differential, we wanted to see whether we could capture the interplay between transcriptional and translational features in a more quantitative way. Theoretical models imply that both transcription and translation bursts lead to an increase in noise strength [17,20,26,52,53] - equivalent to the Fano factor as defined by $F=\sigma^{2} / \mu=C V^{2} \cdot \mu$ (note that for a Poissonian process $F=1$, while a process with $F>1$, i.e. $C V^{2}>1 / \mu$, is considered to be noisy). Specifically, if $B(g)$ is the transcription burst size of gene $g$ and $C(g)$ is the translation burst size then, ignoring any other noise contributors, noise strength can be approximated as the product $B(g) \cdot C(g)$ (see Materials and Methods for additional derivation). Here, we would like to capture the relative contributions of the $B(g)$ and $C(g)$ components to the noise strength.

We focused on the tAI measure of codon usage as a translationrelated feature that correlated most strongly with noise differential in our study. The correlation with $5^{\prime}$ UTR RNA structure was considerably weaker, so we did not consider it in this analysis. Defining noise strength amplification defined as a fold-increase of noise strength for constant protein abundance, we wanted to capture the interplay between the noise strength amplification that can be attributed to increased tAI and the noise strength amplification that can be attributed to presence of a TATA box. The latter relation was identified in previous studies as one of the most prominent transcriptional noise contributors [26,54,55]. We note that noise strength amplification (a concept similar to the noise residual [32]) implies an increase of noise differential. This is explained more naturally in terms of theoretical models (see Materials and Methods).

To estimate noise strength amplification due to TATA box presence and compare it to noise strength amplification attributed to high tAI, we devised two complementary approaches. First, we divided all nonribosomal genes into three groups: TATA genes with high tAI, non-TATA genes with high tAI, and non-TATA genes with low tAI (Figure 3a). All three groups overlap on a certain abundance interval (contain a subgroup of proteins with similar abundance), allowing us to estimate noise strength amplification. Specifically, the average ratio of Fano factor values for TATA genes with high tAI to those for non-TATA genes with high tAI provides an estimate of the noise strength amplification that can be associated with the TATA box to be $\beta=1.27 \pm 0.07$ (YEPD). Along the same lines, the average ratio of Fano factors for non-TATA genes with high tAI to those for non-TATA genes with 

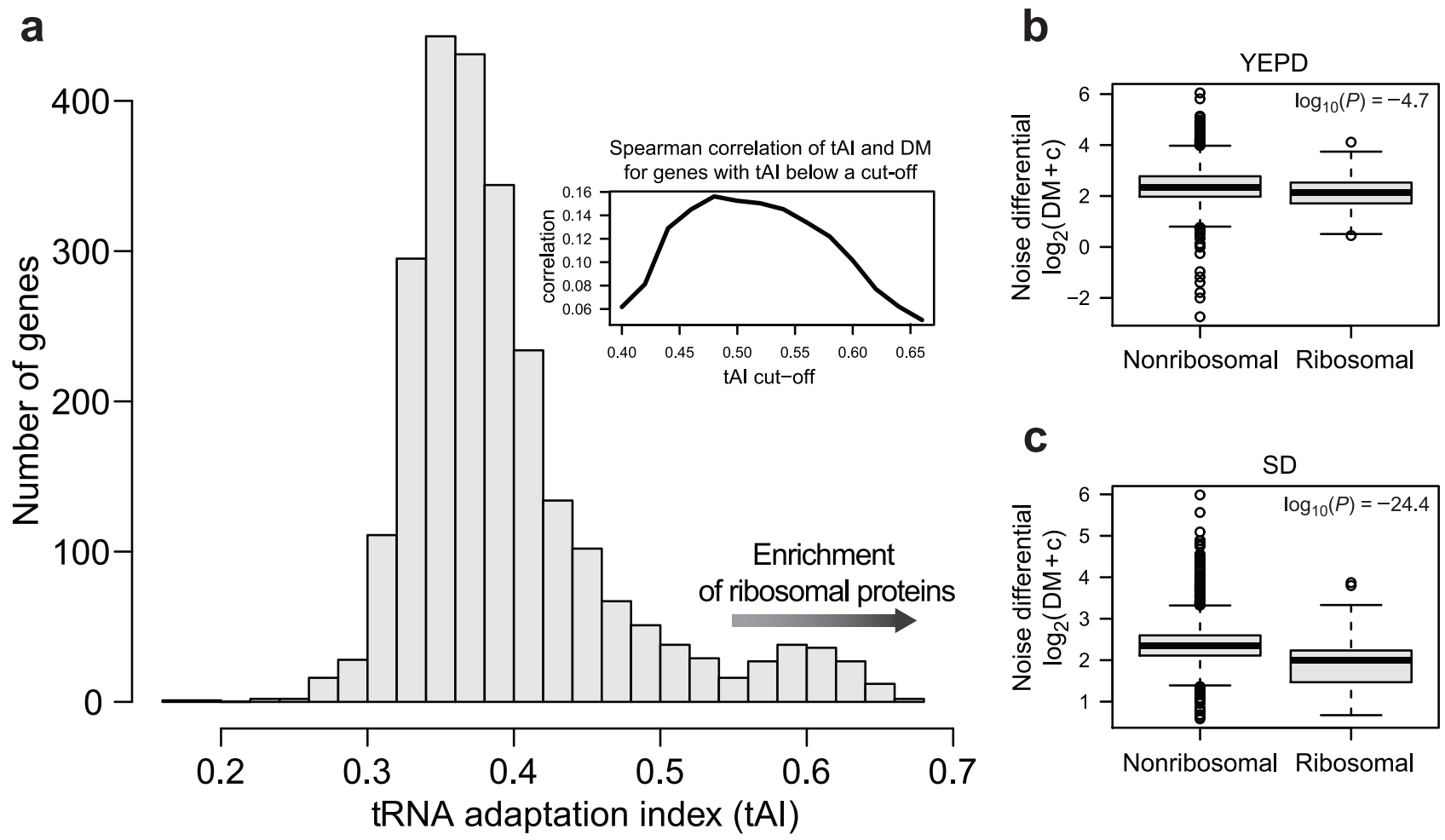

d

\begin{tabular}{|l|rr|rr|rr|}
\hline \multirow{2}{*}{ Protein groups } & \multicolumn{2}{|c|}{$\begin{array}{c}\text { Spearman correlation } \\
\text { between tAl and DM }\end{array}$} & \multicolumn{2}{|c|}{$\log _{10}(P$-value $)$} & \multicolumn{2}{|c|}{ Number of proteins } \\
\cline { 2 - 7 } & \multicolumn{1}{|c|}{ YEPD } & SD & YEPD & SD & YEPD & SD \\
\hline All proteins & 0.070 & 0.049 & -3.0 & -1.6 & 2205 & 2054 \\
Nonribosomal & $\mathbf{0 . 1 5 0}$ & $\mathbf{0 . 1 4 4}$ & -11.1 & -9.6 & 2051 & 1926 \\
Ribosomal & -0.413 & -0.420 & -7.0 & -7.0 & 154 & 148 \\
\hline
\end{tabular}

Figure 1. (a) Histograms of tRNA adaptation index (tAl) scores of budding yeast genes shows a long tail of high tAl values that is highly enriched in ribosomal genes (98 out of 153 genes with $\mathrm{tAl}>0.55$, binomial test $p$-value $<\mathrm{e}-74$ ). Inset: Spearman correlation between tAl and DM increases at a wide range of high tAl cut-off values. (b,c) Ribosomal and nonribosomal genes have different distributions of noise differentials (DM) in both YEPD and SD media - the ribosomal genes are significantly less noisy. For graphing purpose the DM values are shifted by constant $c=5$ prior taking the logarithm. (d) Spearman correlation between tAI and noise differential (DM) for the whole dataset, including nonribosomal and ribosomal proteins in YEPD and SD media.

doi:10.1371/journal.pcbi.1002644.g001

low tAI provides an estimate $\alpha=1.19 \pm 0.02$ (YEPD) of noise strength amplification that can be associated to high tAI values. The estimates based on data in SD medium are similar (Supplementary Table S3). This indicates that, on average, the noise strength amplification that accompanies high tAI values is comparable to the noise strength amplification that accompanies the presence of a TATA box.

As an alternative approach to estimate $\beta$, we divided the genes into two groups: genes containing a TATA box and genes without the TATA box. We looked at noise strength as a function of codon usage (Figure 3b) and directly compared noise strength for TATA and non-TATA genes at the same codon usage values. If the TATA box leads to noise strength amplification $\beta$, then the trend for the relation between codon usage and noise strength for TATA genes should be related to the trend for non-TATA genes by a multiplicative factor $\beta$. This is indeed what we observed (Figure 3b). Assuming that TATA and non-TATA genes with the same tAI are, on average, under the influence of the same extrinsic noise, then this graph provides an estimate of $\beta=1.23 \pm 0.07$ (YEPD), well within the error bars of the previous estimation. This consistency, and the fact that the noise strength amplification observed in Figure $3 \mathrm{~b}$ is consistent over the full range of codon usage, including low codon usage genes that are typically not very abundant, suggests that this estimate is not affected by extrinsic noise.

\section{Discussion}

We examined the deviations from the general trend where expression noise scales with protein abundance. In principle, all processes involved in gene expression can contribute to such a deviation. However, pinpointing the relative contributions of these components is nontrivial. Our study clearly demonstrated a relation between genomic features associated with the translational 
a

\section{Codon usage}

$P$-value of Spearman correlation for nonribosomal proteins YEPD: $\log _{10}(P)=-11.1 \quad$ SD: $\log _{10}(P)=-9.6$

Wilcoxon rank-sum test between noise groups $\log _{10}(P)=-35.2$

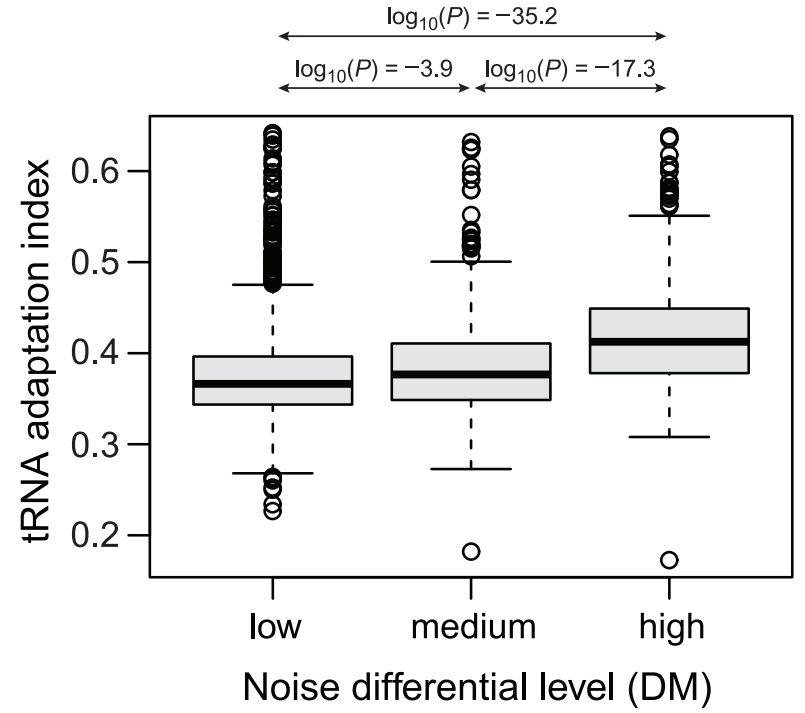

b

\section{5'UTR structure}

$P$-value of Spearman correlation for nonribosomal proteins YEPD: $\log _{10}(P)=-3.2 \quad$ SD: $\log _{10}(P)=-8.2$

Wilcoxon rank-sum test between noise groups

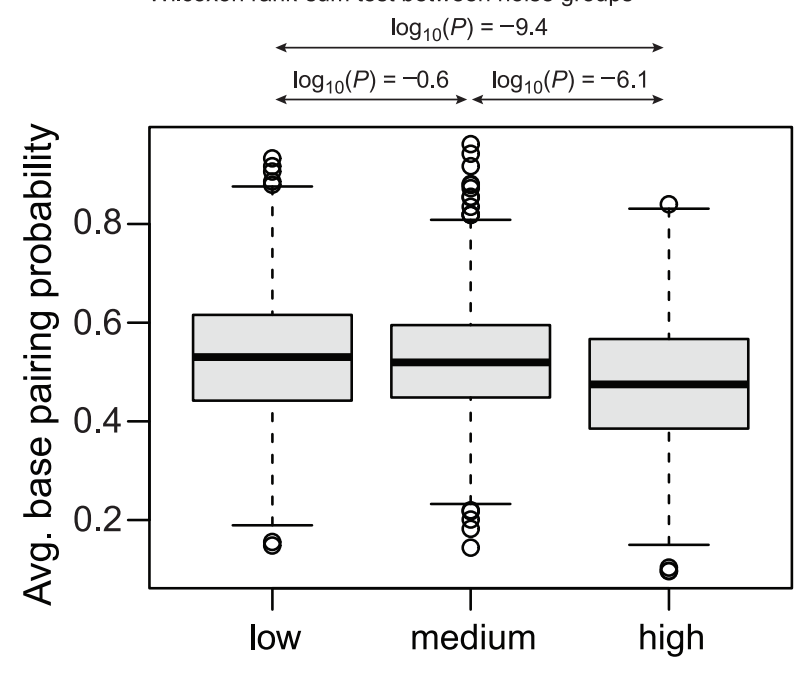

Noise differential level (DM)

Figure 2. Comparison of translation related mRNA features: (a) codon usage (tRNA adaptation index and (b) average base pairing probability at the 5' UTR mRNA structure for different noise differential levels. The nonribosomal genes were subdivided into three groups: low, medium and high noise genes, according to their noise differential levels in YEPD and SD media. doi:10.1371/journal.pcbi.1002644.g002

process and intrinsic noise. High codon usage and low content of 5' UTR secondary structure correlated with increased expression noise differential. The relative contribution of transcription and translation features to noise varied for different gene groups. In particular, high codon usage ribosomal genes are characterized by low noise differential. This heterogeneity is probably a primary reason behind the difficulties in uncovering the interplay between various contributors.

We also performed an initial estimation of the noise strength amplification associated with TATA box presence and noise a

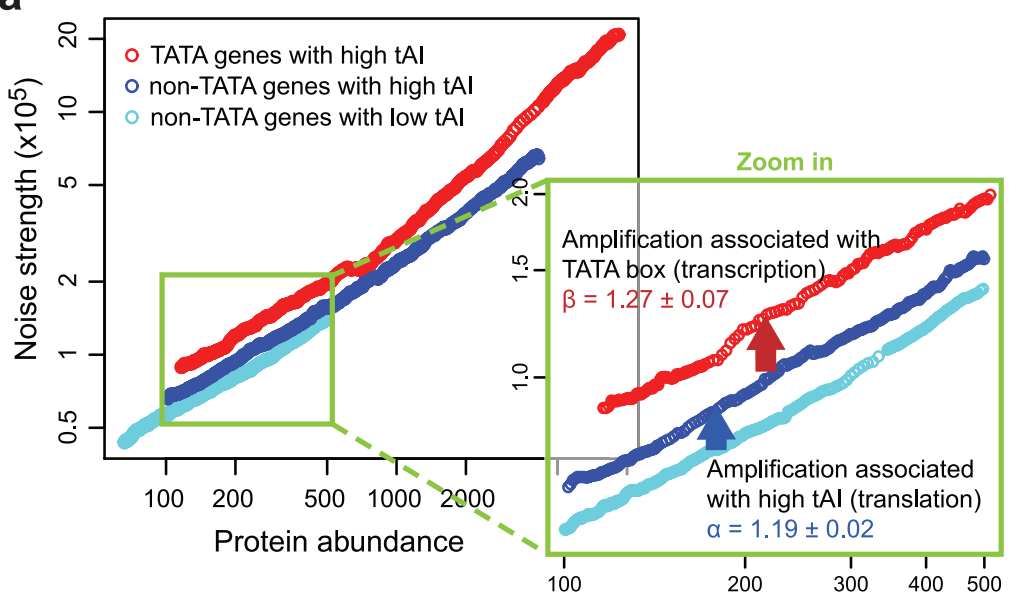

b

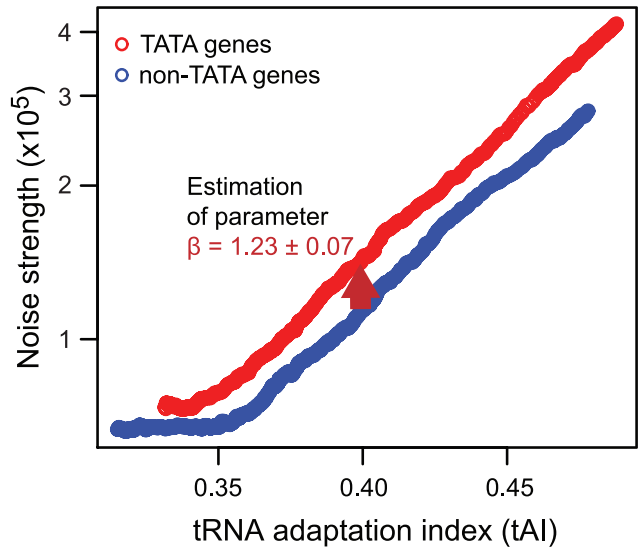

Figure 3. Decomposing noise strength amplification into TATA and tAl associated components. (a) The trend lines for the relation between protein abundance and noise strength (YEPD medium) in three groups of genes: TATA genes with high tAI (red), non-TATA genes with high tAl (blue) and non-TATA genes with low tAl (cyan). High and low tAI mean upper and lower tertile of tAl distribution, respectively. The abundance region where all three trend lines overlap is enlarged. The shift between TATA and non-TATA genes, both with high tAl, represents an amplification associated with the TATA box (transcription feature), $\beta=1.27 \pm 0.07$, while the shift between non-TATA genes with high and low tAl represents an amplification associated with high codon usage (translation feature), $\alpha=1.19 \pm 0.02$. (b) The trend for the noise strength (YEPD medium) as a function of codon usage efficiency (tAl) for TATA genes (red) and non-TATA genes (blue). The shift between these two trend lines provides an alternative estimate of $\beta$, representing the impact of the TATA box on noise strength.

doi:10.1371/journal.pcbi.1002644.g003 
strength amplification that accompanies genes with high codon usage. Surprisingly, on average, the amplification of noise strength that accompanies high codon usage is comparable to the amplification that accompanies TATA box.

While precise factoring of intrinsic noise into its constitutive components requires additional experimental data, the approach developed in this study allowed us to provide initial estimates of the relative contributions of transcriptional and translational noise factors. The noise factoring strategy that we introduced in this study is general and can be used to measure the relative impact of other noise factors.

\section{Materials and Methods}

\section{Data sources}

Ribosomal genes. The list of ribosomal genes was downloaded from the website of the SGD (Saccharomyces Genome Database) project under the GO term "Structural constituent of ribosome" (GO: 0003735, http://www.yeastgenome.org/cgi-bin/ GO/goTerm.pl?goid = 3735). It consists of 224 manually curated genes.

TATA -genes. The list of genes with TATA-containing and TATA-less promoters was obtained from a study by Basehoar et al. [56].

Noise and groups. We used single-cell profiling measurements of protein abundance and its cell-to-cell variations in $S$. cerevisiae, provided by Newman et al. [29]. The experimenters measured steady-state protein levels for cells grown in rich (YEPD) and minimal (SD) media. The $D M$ values (referred here as noise differentials) were used to quantify gene-specific noise levels.

In this study, we classified genes according to noise level in both YEPD and SD media. We subdivided the genes into three groups using somewhat arbitrary thresholds for noise level (DM):

- high noise genes - genes with high noise level in at least one medium, i.e. $\mathrm{DM}_{\mathrm{SD}}>4$ or $\mathrm{DM}_{\mathrm{YEPD}}>4$;

- medium noise genes - genes with medium noise level in at least one medium but without high noise level in any medium, i.e. $1<\mathrm{DM}_{\mathrm{SD}} \leq 4$ or $1<\mathrm{DM}_{\mathrm{YEPD}} \leq 4$ and $\mathrm{DM}_{\mathrm{SD}} \leq 4 ; \mathrm{DM}_{\mathrm{YEPD}} \leq 4$;

- low noise genes - genes with low noise level in any medium where it was measured but without medium or high noise level in any medium, i.e. $\mathrm{DM}_{\mathrm{SD}} \leq 1$ and $\mathrm{DM}_{\mathrm{YEPD}} \leq 1$.

This classification resulted in 1432 genes in the low level, 571 genes in the medium level, and 315 genes in the high level group (total 2328 genes). Note that all ribosomal genes were removed from this analysis.

\section{Computing codon usage}

We measured the translation efficiency by the tAI score, which takes into account the availability of tRNA for each codon, and the efficiency of the codon-anticodon coupling. We followed the definition of Tuller et al. [57].

\section{Computing base pair probability}

All $S$. cerevisiae gene sequences with 100 bases upstream were downloaded from the UCSC genome browser [58] (June 2008 genome assembly of $S$. cerevisiae). We used the RNAplfold program from the Vienna RNA package [59] to compute the base pair probabilities of RNA secondary structure for all sequences in our dataset. RNAplfold computes local pair probabilities; the probabilities are averaged over all windows of given size $\mathrm{L}$ that contain the base pair. We ran RNAplfold with a few different window sizes in the range of 100-300 nucleotides and obtained quite similar results. The results presented here are based on a window size of 150 nucleotides. The composite pairing probability profile was built by averaging base pairing probabilities over all genes for each position in the profile.

\section{Noise model}

Assuming that protein abundance is characterized by two parameters - the mean number of protein production bursts per cell cycle, and the mean number of proteins produced per burst Friedman et al. established the correspondence between these parameters and steady-state distribution. In a system where both transcription bursts and translation bursts are assumed to contribute to the total burst in protein abundance, noise strength $F(g)$, can be decomposed further into transcriptional and translational components. Specifically, if $B(g)$ is the transcription burst size of gene $g$ and $C(g)$ is the number of proteins translated from one mRNA molecule then, ignoring any other noise contributors, the noise strength $F(g)$ can be approximated as $B(g) \cdot C(g)$.

As an alternative derivation, following Raser et al. [26] (equation [6] SOM), we simplified the expression for noise strength as

$$
F \approx 1+E_{m \rightarrow p}+E_{m \rightarrow p} \frac{k_{m} \gamma_{a}}{\left(k_{a}+\gamma_{a}\right)^{2}}
$$

where $E_{m \rightarrow p}$ is the average number of proteins produced from a single mRNA molecule, $k_{\alpha}$ is the promoter activation rate, $k_{m}$ is the RNA production rate, and $\gamma_{\alpha}$ is the promoter closing rate. Assuming that the protein production rate is proportional to codon usage $C(g)$ and that the transcription-related noise strength is attributed to a transcription burst size $B(g)$ we have

$$
F \approx 1+E_{m \rightarrow p}\left(1+\frac{k_{m} \gamma_{a}}{\left(k_{a}+\gamma_{a}\right)^{2}}\right) \approx 1+C(g) B(g) .
$$

\section{Noise trends and computing noise strength amplification}

We used a trend line to smooth out fluctuations in the noise data and to show an underlying pattern more clearly. To compute the trend line, we used the moving average method with overlapping windows of fixed number of genes. We used two different window sizes depending on the size of the gene group: 100 and 300 genes for data in Figure 3a and 3b, respectively. To estimate noise strength amplification (parameters $\alpha$ and $\beta$ ), we divided the interval where trend lines of considered gene groups overlap into bins, and for each bin we computed the ratio of mean trend values between each pair of gene groups. As an estimate of each parameter we took the average value of computed ratios.

\section{Computational platforms}

All calculations and statistical analyses were performed using the R statistical environment (http://www.r-project.org). Scripts were written in the Python programming language (http://www. python.org/).

\section{Supporting Information}

Table S1 $P$-values for Wilxocon tests performed on the original data groups and on sampled groups. (XLSX)

Table S2 Pairwise Spearman's rank correlation between DM, tAI and 5' UTR structure for nonribosomal genes, and partial correlations controlling for tAI, 5' UTR and TATA presence. (XLSX) 
Table S3 Estimates of noise strength amplification associated with the TATA box (parameter $\alpha$ ) and the tRNA adaptation index (parameter $\beta$ ), based on data from YEPD and SD media. (XLSX)

\section{Acknowledgments}

The authors thank Daniela Ganelin for editorial assistance.

\section{References}

1. Elowitz MB, Levine AJ, Siggia ED, Swain PS (2002) Stochastic gene expression in a single cell. Science 297: 1183-1186.

2. Raser JM, O'Shea EK (2005) Noise in gene expression: origins, consequences, and control. Science 309: 2010-2013.

3. Kaern M, Elston TC, Blake WJ, Collins JJ (2005) Stochasticity in gene expression: from theories to phenotypes. Nat Rev Genet 6: 451-464.

4. Fraser HB, Hirsh AE, Giaever G, Kumm J, Eisen MB (2004) Noise minimization in eukaryotic gene expression. PLoS Biol 2: e137.

5. Bahar R, Hartmann CH, Rodriguez KA, Denny AD, Busuttil RA, et al. (2006) Increased cell-to-cell variation in gene expression in ageing mouse heart. Nature 441: 1011-1014

6. Lehner B (2008) Selection to minimise noise in living systems and its implications for the evolution of gene expression. Mol Syst Biol 4: 170 .

7. Becskei A, Serrano L (2000) Engineering stability in gene networks by autoregulation. Nature 405: 590-593.

8. Acar M, Becskei A, van Oudenaarden A (2005) Enhancement of cellular memory by reducing stochastic transitions. Nature 435: 228-232.

9. Chang HH, Hemberg M, Barahona M, Ingber DE, Huang S (2008) Transcriptome-wide noise controls lineage choice in mammalian progenitor cells. Nature 453: 544-547.

10. Janes KA, Wang C-C, Holmberg KJ, Cabral K, Brugge JS (2010) Identifying single-cell molecular programs by stochastic profiling. Nat Meth 7: 311-317.

11. Becskei A, Seraphin B, Serrano L (2001) Positive feedback in eukaryotic gene networks: cell differentiation by graded to binary response conversion. EMBO J 20: $2528-2535$.

12. Rao GV, Wolf DM, Arkin AP (2002) Control, exploitation and tolerance of intracellular noise. Nature 420: 231-237.

13. Kramer BP, Fussenegger M (2005) Hysteresis in a synthetic mammalian gene network. Proc Natl Acad Sci U S A 102: 9517-9522.

14. Guido NJ, Wang X, Adalsteinsson D, McMillen D, Hasty J, et al. (2006) A bottom-up approach to gene regulation. Nature 439: 856-860.

15. Zhang Z, Qian W, Zhang J (2009) Positive selection for elevated gene expression noise in yeast. Mol Syst Biol 5: 299.

16. Wang Z, Zhang J (2011) Impact of gene expression noise on organismal fitness and the efficacy of natural selection. Proc Natl Acad Sci U S A 108: E67-76.

17. Thattai M, van Oudenaarden A (2001) Intrinsic noise in gene regulatory networks. Proc Natl Acad Sci U S A 98: 8614-8619.

18. Osella M, Bosia C, Cora D, Caselle M (2011) The role of incoherent microRNA-mediated feedforward loops in noise buffering. PLoS Comput Biol 7: e1001101.

19. Kittisopikul M, Suel GM (2010) Biological role of noise encoded in a genetic network motif. Proc Natl Acad Sci U S A 107: 13300-13305.

20. Rodriguez Martinez M, Soriano J, Tlusty T, Pilpel Y, Furman I (2010) Messenger RNA fluctuations and regulatory RNAs shape the dynamics of a negative feedback loop. Phys Rev E Stat Nonlin Soft Matter Phys 81: 031924.

21. Jothi R, Balaji S, Wuster A, Grochow JA, Gsponer J, et al. (2009) Genomic analysis reveals a tight link between transcription factor dynamics and regulatory network architecture. Mol Syst Biol 5: 294.

22. Li J, Min R, Vizeacoumar FJ, Jin K, Xin X, et al. (2010) Exploiting the determinants of stochastic gene expression in Saccharomyces cerevisiae for genome-wide prediction of expression noise. Proc Natl Acad Sci U S A 107: 10472-10477.

23. Swain PS, Elowitz MB, Siggia ED (2002) Intrinsic and extrinsic contributions to stochasticity in gene expression. Proc Natl Acad Sci U S A 99: 12795-12800.

24. Hilfinger A, Paulsson J (2011) Separating intrinsic from extrinsic fluctuations in dynamic biological systems. Proc Natl Acad Sci U S A 108: 12167-12172.

25. Maheshri N, O'Shea EK (2007) Living with noisy genes: how cells function reliably with inherent variability in gene expression. Annu Rev Biophys Biomol Struct 36: 413-434.

26. Raser JM, O'Shea EK (2004) Control of Stochasticity in Eukaryotic Gene Expression. Science 304: 1811-1814.

27. Maamar H, Raj A, Dubnau D (2007) Noise in Gene Expression Determines Cell Fate in Bacillus subtilis. Science 317: 526-529.

28. Mao C, Brown CR, Falkovskaia E, Dong S, Hrabeta-Robinson E, et al. (2010) Quantitative analysis of the transcription control mechanism. Mol Syst Biol 6: 431.

29. Newman JR, Ghaemmaghami S, Ihmels J, Breslow DK, Noble M, et al. (2006) Single-cell proteomic analysis of $\mathrm{S}$. cerevisiae reveals the architecture of biological noise. Nature 441: 840-846.

30. Ozbudak EM, Thattai M, Kurtser I, Grossman AD, van Oudenaarden A (2002) Regulation of noise in the expression of a single gene. Nat Genet 31: 69-73.

\section{Author Contributions}

Conceived and designed the experiments: TMP. Performed the experiments: RS DW JZ. Analyzed the data: RS DW JZ DL YP TMP. Wrote the paper: RS DW JZ DL YP TMP.
31. Blake WJ, M KA, Cantor CR, Collins JJ (2003) Noise in eukaryotic gene expression. Nature 422: 633-637.

32. Bar-Even A, Paulsson J, Maheshri N, Carmi M, O'Shea E, et al. (2006) Noise in protein expression scales with natural protein abundance. Nat Genet 38: 636-643.

33. Golding I, Paulsson J, Zawilski SM, Cox EC (2005) Real-time kinetics of gene activity in individual bacteria. Cell 123: 1025-1036.

34. Raj A, Peskin CS, Tranchina D, Vargas DY, Tyagi S (2006) Stochastic mRNA synthesis in mammalian cells. PLoS Biol 4: e309.

35. Suter DM, Molina N, Gatfield D, Schneider K, Schibler U, et al, (2011) Mammalian Genes Are Transcribed with Widely Different Bursting Kinetics. Science 332: 472-474.

36. Cai L, Friedman N, Xie XS (2006) Stochastic protein expression in individual cells at the single molecule level. Nature 440: 358-362.

37. Silander OK, Nikolic N, Zaslaver A, Bren A, Kikoin I, et al. (2012) A GenomeWide Analysis of Promoter-Mediated Phenotypic Noise in Escherichia coli. PLoS Genet 8: e1002443.

38. Pilpel Y (2011) Noise in biological systems: pros, cons, and mechanisms of control. Methods Mol Biol 759: 407-425.

39. Grantham R, Gautier C, Gouy M, Jacobzone M, Mercier R (1981) Codon catalog usage is a genome strategy modulated for gene expressivity. Nucleic Acids Res 9: r43-74.

40. Bennetzen JL, Hall BD (1982) Codon selection in yeast.J Biol Chem 257: 3026-3031.

41. Gouy M, Gautier C (1982) Codon usage in bacteria: correlation with gene expressivity. Nucleic Acids Res 10: 7055-7074.

42. Taniguchi Y, Choi PJ, Li GW, Chen H, Babu M, et al. (2010) Quantifying E. coli proteome and transcriptome with single-molecule sensitivity in single cells. Science 329: 533-538.

43. Kertesz M, Wan Y, Mazor E, Rinn JL, Nutter RC, et al. (2010) Genome-wide measurement of RNA secondary structure in yeast. Nature 467: 103-107.

44. Pestova TV, Kolupaeva VG (2002) The roles of individual eukaryotic translation initiation factors in ribosomal scanning and initiation codon selection. Genes Dev 16: 2906-2922.

45. Ingolia NT, Ghaemmaghami S, Newman JR, Weissman JS (2009) Genomewide analysis in vivo of translation with nucleotide resolution using ribosome profiling. Science 324: 218-223.

46. Kudla G, Murray AW, Tollervey D, Plotkin JB (2009) Coding-sequence determinants of gene expression in Escherichia coli. Science 324: 255-258.

47. de Smit MH, van Duin J (1990) Secondary structure of the ribosome binding site determines translational efficiency: a quantitative analysis. Proc Natl Acad Sci U S A 87: 7668-7672.

48. Ringner M, Krogh M (2005) Folding free energies of $5^{\prime}$-UTRs impact posttranscriptional regulation on a genomic scale in yeast. PLoS Comput Biol 1: e72.

49. dos Reis M, Wernisch L, Savva R (2003) Unexpected correlations between gene expression and codon usage bias from microarray data for the whole Escherichia coli K-12 genome. Nucleic Acids Res 31: 6976-6985.

50. Alhabshan F, Smallhorn JF, Golding F, Musewe N, Freedom RM, et al. (2005) Extent of myocardial non-compaction: comparison between MRI and echocardiographic evaluation. Pediatr Radiol 35: 1147-1151.

51. Gu W, Zhou T, Wilke CO (2010) A universal trend of reduced mRNA stability near the translation-initiation site in prokaryotes and eukaryotes. PLoS Comput Biol 6: e1000664.

52. Friedman N, Cai L, Xie XS (2006) Linking stochastic dynamics to population distribution: an analytical framework of gene expression. Phys Rev Lett 97: 168302.

53. Paulsson J, Ehrenberg M (2000) Random signal fluctuations can reduce random fluctuations in regulated components of chemical regulatory networks. Phys Rev Lett 84: 5447-5450.

54. Blake WJ, Balazsi G, Kohanski MA, Isaacs FJ, Murphy KF, et al. (2006) Phenotypic consequences of promoter-mediated transcriptional noise. Mol Cell 24: 853-865.

55. Mogno I, Vallania F, Mitra RD, Cohen BA (2010) TATA is a modular component of synthetic promoters. Genome Res 20: 1391-1397.

56. Basehoar AD, Zanton SJ, Pugh BF (2004) Identification and distinct regulation of yeast TATA box-containing genes. Cell 116: 699-709.

57. Tuller T, Carmi A, Vestsigian K, Navon S, Dorfan Y, et al. (2010) An evolutionarily conserved mechanism for controlling the efficiency of protein translation. Cell 141: 344-354.

58. Kent WJ, Sugnet CW, Furey TS, Roskin KM, Pringle TH, et al. (2002) The human genome browser at UCSC. Genome Res 12: 996-1006.

59. Bernhart SH, Hofacker IL, Stadler PF (2006) Local RNA base pairing probabilities in large sequences. Bioinformatics 22: 614 615 . 